\title{
High Density Thermomagnetic Recording on Flux Detectable RE-TM Media
}

\author{
Hiroaki Nemoto, Hideki Saga, Hirofumi Sukeda, and Masahiko Takahashi \\ Optical Disk Research Center, Storage Research Department, Central Research Laboratory, Hitachi Lid., \\ 1-280 Higashi-Koigakubo, Kokubunji-shi, Tokyo 185-860I, Japan
}

Flux-detectable and thermomagnetically recordable media were prepared by sputtering amorphous RE-TM alloys. We investigated the recording characteristics of these media at an ultra-high recording density. A laserstrobe magnetic-field modulation recording scheme was applied to these media in order to form much shorter domains than the diameter of the optical spot. We observed the recorded magnetic domains by magnetic field microscopy and found irregular domains. Domains shorter than $150 \mathrm{~nm}$ became collapsed or combined with other domains. To resolve this problem, a heat sink structure was introduced to this media. This structure turned out to be especially effective, and we could record 75-nm-length domains with an Al heat sink layer even if an optical spot with a diameter of $1000 \mathrm{~nm}$ was used. The high-density recording capability of these media was confirmed.

Key words: flux detection, rare-earth transition-metal alloys, thremomagnetic recording, heat-sink structure, magnetic field modulation, magneto-resistive (MR) sensor, magnetic force microscopy

\section{Introduction}

The invention of the magneto-resistive (MR) magneticflux sensor was the most epoch-making event in the field of magnetic recording devices. The MR sensor is more sensitive and more suitable for fine processing than conventional inductive sensor. Based on this sensor, the detection resolution of a read-head has been improved by reducing the shield-gap length and the head flying height, and the signal processing method has been optimized. As a result, recording density has been increasing by $60 \%$ every year, since 1992.

However, existing magnetic recording media encounter a problem at a density of over $20 \mathrm{~Gb} / \mathrm{inch}^{2}$, . As the size of the recording bits decreases, magnetic grains in the recording layer must also become smaller in order to achieve accurate recording. In this case, the thermal fluctuation causes random magnetization reversals which gradually reduce the contrast of recording bits. This domain vanishing phenomena is a serious problem with the magnetic recording media.

Magneto-optical (MO) recording media are expected to be free from such phenomena, because they do not have a grain structure. However, they have not been appropriate for use as a magnetic recording medium. Honda et al. have examined recording characteristics of a ring-type writing head on a perpendicular Pt/Co multi-layer medium. ${ }^{2-3)}$ Large bit transition fluctuations were observed in magnetic field micrographs and the reproduced signal showed a lot of recording noise. This noise is considered to be due to the strong exchangecoupling of the magnetic layer at room temperature. Amorphous rare earth (RE) - transition metal (TM) alloys have also been examined before, ${ }^{4-5}$ which led to the same result.

To draw out the potential of RE-TM alloys as a magnetic recording medium, we proposed using a new recording scheme consisting of thermomagnetic writing and magnetic flux detection. ${ }^{6)}$ This means that we use the MR sensor to detect magnetic domains which are thermomagnetically recorded by using an optical head.

We must verify the capability of ultra-high-density recording over $20 \mathrm{~Gb} /$ inch $^{2}$ in order to take advantage of our scheme. Although it has been shown that $100-\mathrm{nm}$ length domains could be formed on a TbFeCo film with an optical spot diameter of about $1000 \mathrm{~nm}$," adequate conditions to record shorter magnetic domains are not clear. In this paper, the observation result of submicronlength domains recorded by using laser-strobe magnetic field modulation (LS-MFM) method is reported first. Then we describe how to control the recording characteristics.

\section{Experiment and Results}

\subsection{Experimental setup}

In Ref. 6, we demonstrated a flux-detectable and thermomagnetically recordable medium using a $\mathrm{TbFeCo}$ alloy. This medium contains two magnetic layers which are exchange-coupled to each other. According to the result, we prepared two sample disks. Table I lists the structure of the samples.

The recording film is deposited on a polycarbonate (PC) disk substrate by RF magnetron sputtering. The disk substrate was baked at $80^{\circ} \mathrm{C}$ in the vacuum before deposition. It was an usual optical disk replica which

Table 1 Structures of sample disks

\begin{tabular}{|lcc|}
\hline & Disk A & Disk B \\
\hline lubricant (Fomblin) & $3 \mathrm{~nm}$ & $3 \mathrm{~nm}$ \\
\hline protective layer $\left(\mathrm{Si}_{4} \mathrm{~N}_{4}\right)$ & $10 \mathrm{~nm}$ & $10 \mathrm{~nm}$ \\
\hline heat sink layer $(\mathrm{Al})$ & & $40 \mathrm{~nm}$ \\
\hline protective layer $\left(\mathrm{Si}_{3} \mathrm{~N}_{4}\right)$ & & $5 \mathrm{~nm}$ \\
\hline readout layer $(\mathrm{TbDyFeCo})$ & $75 \mathrm{~nm}$ & $35 \mathrm{~nm}$ \\
\hline memory layer $(\mathrm{TbFeCo})$ & $40 \mathrm{~nm}$ & $15 \mathrm{~nm}$ \\
\hline protective layer $\left(\mathrm{Si}_{4} \mathrm{~N}_{4}\right)$ & $60 \mathrm{~nm}$ & $60 \mathrm{~nm}$ \\
\hline disk substrate $(\mathrm{PC})$ & $\cdots$ & $\cdots$ \\
\hline
\end{tabular}


was $0.6-\mathrm{mm}$ thick and had land and groove structure (period: $1200 \mathrm{~nm}$ ). The detailed shape was measured by using an atomic force microscope. The width of the land terrace was about $450 \mathrm{~nm}$. We used TbDyFeCo alloy for the readout layer and $\mathrm{TbFeCo}$ alloy for the memory layer. These magnetic layers were sandwiched by $\mathrm{Si}_{3} \mathrm{~N}_{4}$ protective layers to prevent oxidation of rare earth metals and to protect the entire film mechanically.

Disk $A$ had the same structure as shown in Ref. 6 . As the surface protective layer is as thin as $10 \mathrm{~nm}$, it is easy to detect the leakage magnetic flux. Disk B had a heat sink structure with an Al layer deposited nearby the magnetic layers. Because of the large thermal conductivity of Al metal, heat spreads easily to the Al layer and the temperature of the memory layer decreases quickly after a write pulse irradiated. The protective layer was inserted between the magnetic layers and the Al layer to prevent the mixing of $\mathrm{Al}$ atoms into the magnetic layer.

After the films were deposited, the disks were dipped into a lubricant. The lubricant was a variety of Fomblin manufactured by Ausimont S.p.A. The thickness of the lubricant on the disk surface was about $3 \mathrm{~nm}$, as measured by Fourier-transform infrared spectroscopy (FTIR).

Fig. 1 shows the temperature dependence of saturation magnetization for each magnetic layer. Magnetic properties of magnetic layers did not depend remarkably on the thickness. The saturation magnetization of the readout layer was $260 \mathrm{emu} / \mathrm{cm}^{3}$ at room temperature, which was much larger than that of recording layer (below $100 \mathrm{emu} / \mathrm{cm}^{3}$ ). Large magnetization is important to provide high density flux to be detected by an MR sensor or a magnetic field microscope. The readout layer was strongly exchange-coupled to the memory layer in order to copy out the recorded magnetic domains precisely. And the Curie temperature of the readout layer was lower than that of the memory layer. In the thermomagnetic writing process, magnetic domains are formed on the memory layer first and copied to the readout layer after the film temperature falls.

Thermomagnetic writing and optical playback was tested as in a usual MO disk drive by irradiating an incident beam from the substrate side. LS-MFM recording was performed to test the recording

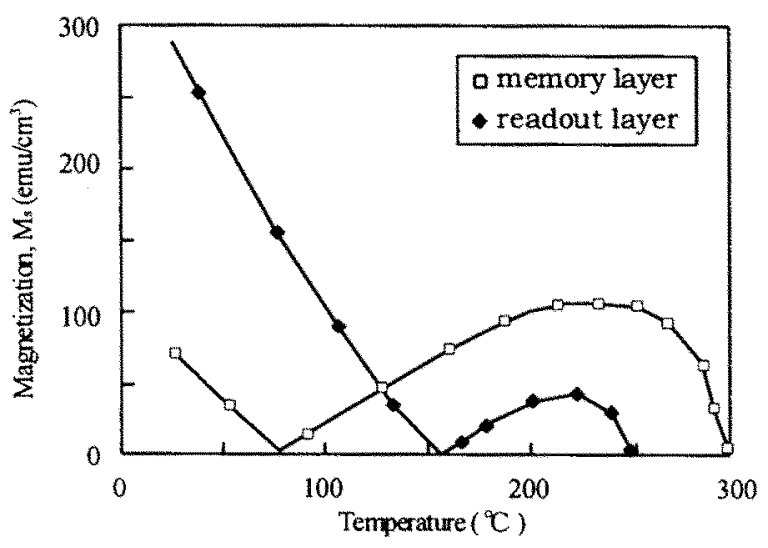

Fig. 1 Magnetization dependence on temperature

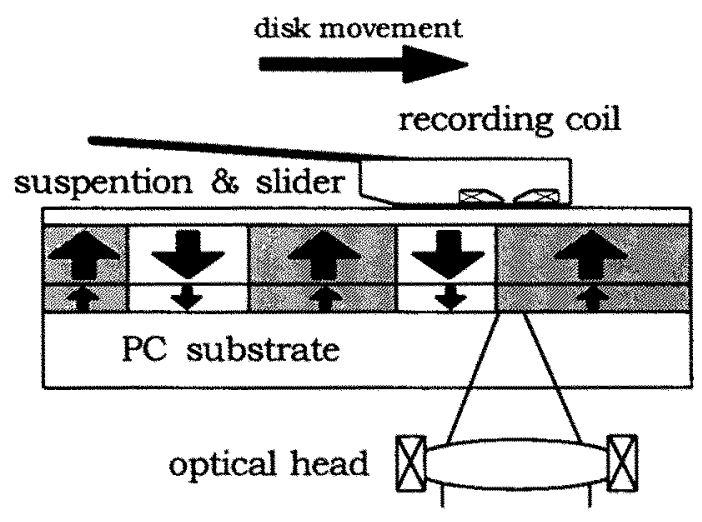

Fig. 2 Setup of the disk tester

characteristics at a high linear density. The setup of our disk tester is shown in Fig. 2 . While writing and reading, disks were rotated at a constant linear velocity of $4 \mathrm{~m} / \mathrm{s}$. A small recording coil was mounted on a flying slider for quick modulation of the magnetic field. The flying height of the slider is about 15 micron. Laser pulses of $50 \%$ duty were irradiated continuously. The absolute peak value of magnetic field was adjusted to be $200 \mathrm{Oe}$ and peak-to-peak switching time was about 5 ns.

\subsection{Breakdown of domain formation}

Conventional magneto-optical media use TbFeCo alloys of compensation compositions. These alloys are advantageous for thermomagnetic writing, but insufficient for magnetic flux detection because of their low flux density at room temperature.

The readout layer of our flux-detectable media makes it easy to investigate domains directly by detecting magnetic flux. Disk $A$ in Fig. 1 is furnished with only a thin surface protective layer and is particularly suitable for flux-detection. We carried out the observation of the magnetic domains on disk $A$ with a magnetic force microscope.

Fig. 3 shows magnetic force micrographs of continuously written magnetic domains. The pulse interval time $T_{w}$ is a reciprocal of the system clock. A unit length $L_{w}$ of a recording domain is determined by multiplying $T_{w}$ by the linear velocity of disk rotation. To write ' $n \cdot T_{w}$ ' domains, $n$ laser pulses are irradiated with magnetic field holding still. So the length is $n \cdot L_{w}$.

Though domains were recorded on the land area, they were slightly extended to the groove area because of the relatively strong irradiation. It was confirmed that short domains down to $200 \mathrm{~nm}$ were well recorded on Disk A as well as on conventional MO media. Some distortion of the domains was attributed to the random motion of the domain walls (DW), particularly at the both edges of the land area. It was remarkable that the larger domains did not have small, reversed-magnetization parts inside at all, as do domains on the magnetic recording media composed of micro-crystalline $\mathrm{CoCr}(\mathrm{Ta})$. 


\begin{tabular}{|c|c|c|}
\hline $1 \mu \mathrm{m}$ & $\begin{array}{c}\mathrm{T}_{\mathrm{w}}=25 \mathrm{~nm} \\
\left(\mathrm{~L}_{\mathrm{w}}=100 \mathrm{~nm}\right)\end{array}$ & $\begin{array}{c}\mathrm{T}_{\mathrm{w}}=18.75 \mathrm{~nm} \\
\left(\mathrm{~L}_{\mathrm{w}}=75 \mathrm{~nm}\right)\end{array}$ \\
\hline $8 \mathrm{~T}_{\mathrm{w}}$ & & f. \\
\hline $4 \mathrm{~T}_{\mathrm{w}}$ & & \\
\hline $2 \mathrm{~T}_{\mathrm{w}}$ & & \\
\hline $1 T_{w}$ & to & 2. \\
\hline
\end{tabular}

Fig. 3 Magnetic force micrographs of recorded magnetic domains for disk A. Lw is the laser pulse period in terms of displacement.

As the domains got shorter than $150 \mathrm{~nm}$, DW became disordered suddenly. We can barely discriminate 100$\mathrm{nm}$ and 75-nm domains in Fig. 3. There are two possibilities that can cause this kind of phenomena. The one is the breakdown of the function of copying the magnetic domains to the readout layer. The other is the problem of domain formation while recording.

\subsection{Thermal property and recording characteristics}

In order to clarify what the cause is, we have to examine the domains in the memory layer optically from the substrate side. Although the resolution of magnetooptical detection is severely limited by optical resolution, such an irregular magnetic state should be reflected in the behavior of noise in a lower frequency region.

Fig. 4 shows reproduced signals of disk $A$ and $B$. Both disks were recorded by LS-MFM. The lengths of written domains were $100 \mathrm{~nm}, 200 \mathrm{~nm}, 400 \mathrm{~nm}$, and $800 \mathrm{~nm}$, respectively. For an optical spot with a diameter of 1000 nm, signals from small magnetic domains inside the optical spot interfered with each other and degrade the total magneto-optical signal-to-noise ratio. The signals from a series of $100-\mathrm{nm}$ and $200-\mathrm{nm}$ domains were averaged to zero, though $800-\mathrm{nm}$ domains was detected clearly.

The difference between disk A and disk B was obvious, particularly for signals from $100-\mathrm{nm}$ domains. The signal from disk A was noisier than disk B. Of course, this signal state was caused by the domains shown in Fig. 3. Moreover, the average signal level from $100-\mathrm{nm}$ domains of disk A was rather lower than that of disk B. This means that on average there are more collapsed domains than expanded or united ones.

Fig. 5 shows the relation between recorded domain length and the increment of low frequency noise level (at 2 $\mathrm{MHz}$ ) from the initialized state. The noise intensity increased rapidly below a domain length of $200 \mathrm{~nm}$ for disk A. This result coincides with the result of Fig. 3,
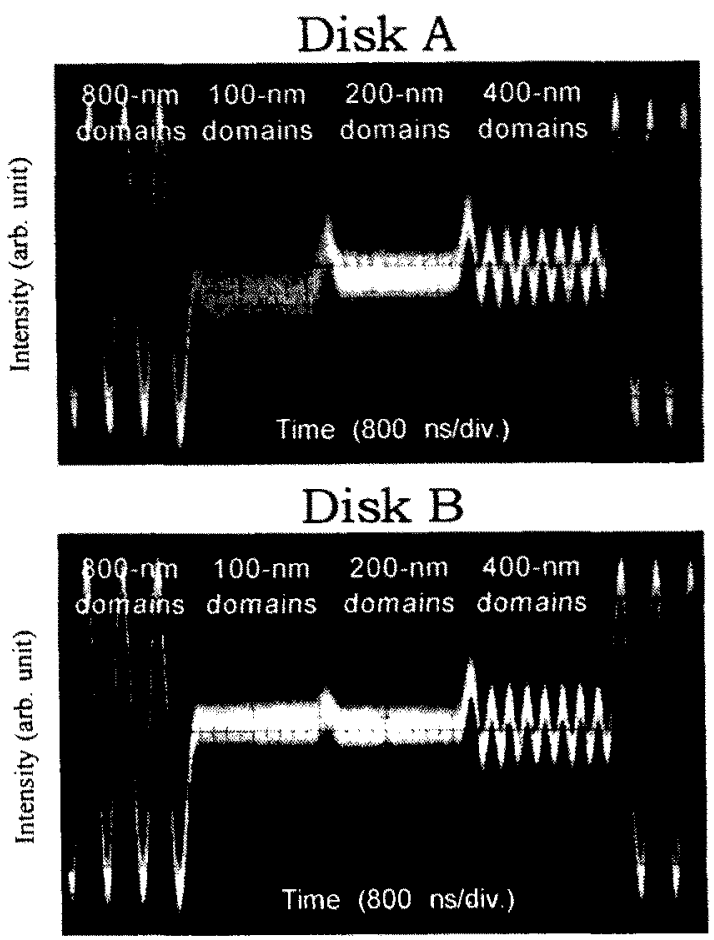

Fig. 4 Magneto-optical reproduced signals for disk A and B

which means that domains of the same quality were formed in both the memory and readout layers. Now we believe that the exchange-coupling is strong enough to ensure accurate copying of the magnetic domains.

After all, we suspect that the DW formation process became out of control while or after the laser was irradiated in disk A. The DW formation is good in case of longer domains, so the frequent reversal of the magnetic field has relation to the breakdown.

In contrast, the signals from disk B showed a steady level and low deviation, which indicates alternate domains were recorded stably. This result suggest that disk B is superior to disk $A$ in controlling the DW behavior. Because the thickness dependence of magnetic layers is

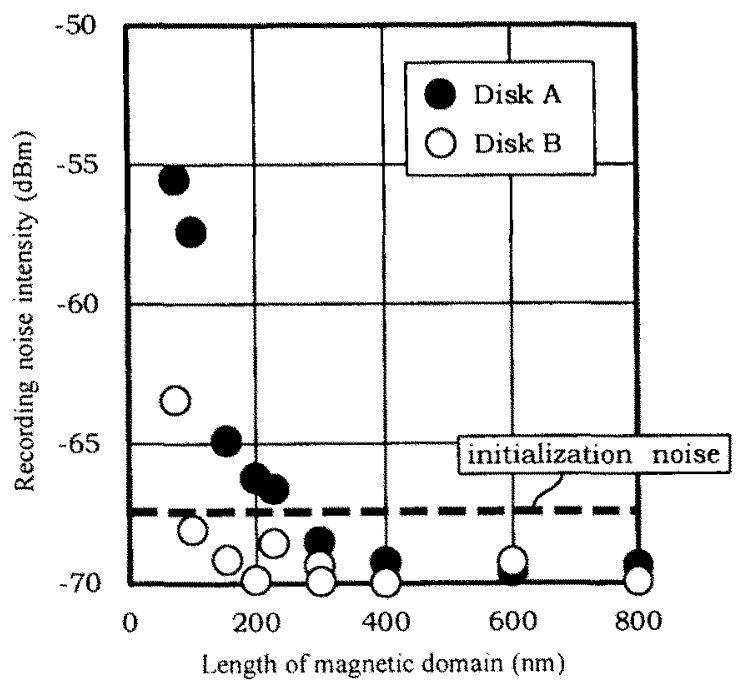

Fig. 5 Domain length v.s. noise level. Recording noise intensity is measured at $2 \mathrm{MHz}$ 
negligible, disk A and disk B are similar to each other magnetically. The reason for this difference is the heat sink structure of disk B. To make sure of the effect of this structure, we examined the thermal behavior of both disks in a computer simulation.

Fig. 6 shows calculated temperature transitions at the position where DW should be fixed. DW is settled once at the position where the memory layer gets heated to $300^{\circ} \mathrm{C}$ at $0 \mathrm{~ns}$. (note that the Curie temperature of the memory layer is $300^{\circ} \mathrm{C}$ ). As shown in the figure, the temperature stays around $250^{\circ} \mathrm{C}$ to $300^{\circ} \mathrm{C}$ for disk $\mathrm{A}$ after that. If the recording magnetic field is reversed, it is difficult to freeze DW in such a situation. An Al heat sink layer is very effective for this. In disk $B$, the temperature goes down to $150^{\circ} \mathrm{C}$ rapidly after recording. This improves DW controllability and makes it possible to form 100-nm length domains without increasing the noise. It was clarified that the design of the thermal structure becomes more important for ultra-high density recording.

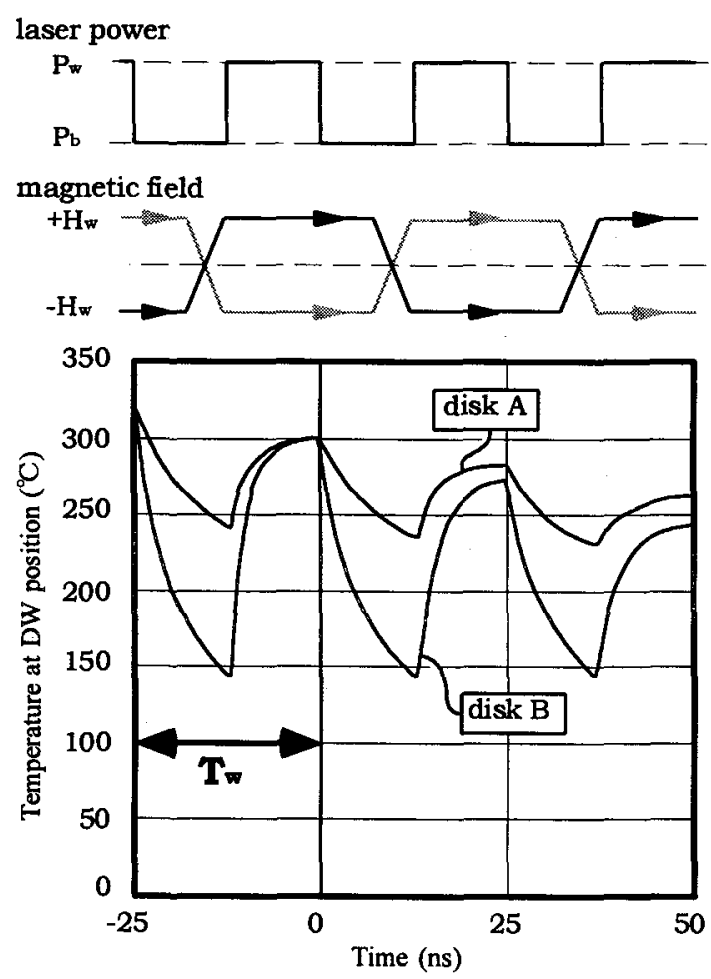

Fig. 6 Calculated temperature change at the DW position. The period of the pulse irradiation ( $\left.T_{w}\right)$ is $25 \mathrm{~nm}$ and the linear velocity is $4 \mathrm{~m} / \mathrm{s}$ which is the condition that $100-\mathrm{nm}$ length domains will be formed if magnetic field is reversed as illustrated.

It is supposed DW position is fixed at $0 \mathrm{~ns}$ when the memory layer is heated up to Curie Temperature of the memory layer $\left(300^{\circ} \mathrm{C}\right)$ for the last time.

\section{Summary}

In this review, we have tried to form very short domains by using LS-MFM. Magnetic field microscopy reveals that the media which we described in Ref. 6 proves not to be suitable for high-density recording. However with a heat sink structure, high-density recording over 20 $\mathrm{Gb} /$ inch $^{2}$ can be achieved.

We have succeeded in reading Disk $A$ by using an MR head at a higher resolution than that of the optical head as described in Ref. 6., so the feasibility of the read/write scheme has been confirmed. But disk B is not suitable for reading with the MR head because the Al heat sink layer peeled off easily. To read Disk $B$, the film structure must be modified.

Betzig et al. has demonstrated that SNOM optics can be used to form magnetic domains with dimensions as small as $60 \mathrm{~nm}^{8}$ ) Therefore our method is one of the most promising ways to achieve a bit density of over 100 $\mathrm{Gb} /$ inch $^{2}$. Our next target is to accurately form domains at a higher density with a smaller optical spot. Now we are planning to irradiate the incident beam from the surface side of the media by using a new type of optical head, which we will develop into a near-field optical head such as one with SIL $^{9)}$ or SNOM optics.

Blue laser is also an option for our technique. Either blue laser sources or near-field optics are indispensable for attaining higher recording densities in the future.

\section{References}

1) P.-L. Lu and S. H. Charap: IEEE Trans. Magn. 30 (1994) 4230

2) Y. Honda, Y. Hirayama, K. Ito, and M. Futamoto: IEEE Trans. Magn. 34 (1998) 1633

3) M. Suzuki, H. Awano, N. Inaba, Y. Honda, and M. Futamoto: J. Magn. Soc. Jpn. 18 Supplement (1994) 451

4) D. Jeanniot and J. Desserre: J. Appl. Phys. 54 (1983) 2820

5) Y. Ohtani, F. Yoshimura, and I. Hatakeyama: IEEE Trans. Magn. 26 (1990) 2292

6) H. Nemoto, H. Saga, H. Sukeda, and M. Takahashi: Jpn. J. Appl. Phys. 38 (1999) to be published

7) T. Shiratori, E. Fujii, Y. Miyaoka, and Y. Hozumi: J. Magn. Soc. Jpn. 22 Supplement No. S2 (1998) 47

8) E. Betzig, J. K. Trautman, R. Wolfe, E. M. Gyorgy, and P. L. Finn: Appl. Phys. Lett. 61 (1992) 142

9) S. M. Mansfield, W. R. Studenmund, G. S. Kino, and K. Osato: Optics Letters 18 (1993) 305 